\title{
Extubação Paliativa em Pacientes Terminais: Revisão Integrativa
}

doi: https://doi.org/10.32635/2176-9745.RBC.2020v66n4.1098

\author{
Palliative Extubation in Terminal Patients: Integrative Review \\ Extubación Paliativa en Pacientes Terminales: Revisión Integradora
}

Beatriz Louro'; Bianca Kemmilly Rodrigues Paiva²; Amanda Estevão

\section{RESUMO}

Introduçáo: $\mathrm{O}$ cuidado paliativo tem como objetivo minimizar o sofrimento tanto do paciente como de seus familiares, por meio de um trabalho multiprofissional. As doenças crônicas não transmissíveis são a principal causa de sofrimento e incapacidade, levando-os aos cuidados paliativos. A fim de amenizar o sofrimento, a extubação paliativa é um procedimento que evita prolongar a morte, por intermédio da retirada de medidas invasivas respiratórias, como a intubação orotraqueal. Objetivo: Realizar uma revisão integrativa e analisar/apresentar o impacto da extubação paliativa em pacientes terminais. Método: Trata-se de um estudo de revisão de literatura e, para sua realização, foi realizada uma busca nas bases de dados PEDro, LILACS e PubMed, utilizando os descritores: airway extubation, palliative care e hospice care. Resultados: Para a realização deste estudo, foram encontrados 41 artigos, sendo 15 relevantes para a revisão. Estudos mostraram que a extubaçáo paliativa é benéfica para o paciente e seus familiares, mesmo que o tempo de morte possa variar de acordo com a doença existente. Conclusáo: Apesar do pequeno número de estudos, foi possível observar que a extubação paliativa se mostrou eficaz no tratamento, mediante relatos de familiares, proporcionando melhor qualidade de vida e uma morte mais tranquila e sem mais sofrimentos.

Palavras-chave: Extubação; Cuidados Paliativos na Terminalidade da Vida/métodos; Futilidade Médica; Doente Terminal.

\section{ABSTRACT}

Introduction: Palliative care aims to minimize the suffering of both patients and their families through a multidisciplinary work. Non-communicable chronic diseases are the leading cause of suffering and disability, leading them to palliative care. In order to alleviate suffering, palliative extubation is a procedure that avoids prolonging death by removing invasive respiratory measures such as orotracheal intubation. Objective: To perform an integrative review and analyze/present the impact of palliative extubation in terminal patients. Method: A literature review study through search performed in the databases PEDro, LILACS and PubMed, using the keywords: airway extubation, palliative care and hospice care. Results: For this study, 41 articles were found, being 15 relevant for the review. Studies have shown that palliative extubation is beneficial for patients and their families, even though the time of death may vary according to the existing disease. Conclusion: Despite the small number of studies, it was possible to observe that palliative extubation proved to be effective in the treatment based in family members reports, ensuring better quality of life and a more peaceful death without further suffering.

Key words: Airway Extubation; Hospice Care/methods; Medical Futility; Terminally III.

\section{RESUMEN}

Introducción: Los cuidados paliativos tienen como objetivo minimizar el sufrimiento de los pacientes y sus familias a través de un trabajo multidisciplinario. Las enfermedades crónicas no transmisibles son la principal causa de sufrimiento y discapacidad que los lleva a los cuidados paliativos. Para aliviar el sufrimiento, la extubación paliativa será un procedimiento que evita prolongar la muerte al eliminar las medidas respiratorias invasivas como la intubación orotraqueal. Objetivo: Realizar una revisión integradora y analizar/presentar el impacto de la extubación paliativa en pacientes con enfermedades terminales. Método: Este es un estudio de revisión de literatura y se realizó una búsqueda en las bases de datos PEDro, LILACS y PubMed, usando las palabras clave: extubación de vías aéreas, cuidados paliativos y cuidados de hospicio. Resultados: Para este estudio, se encontraron 41 artículos, 15 relevantes para la revisión. Los estudios han demostrado que la extubación paliativa es beneficiosa para los pacientes y sus familias, aunque el momento de la muerte puede variar según la enfermedad existente. Conclusión: A pesar del pequeño número de estudios, fue posible observar que la extubación paliativa demostró ser efectiva en el tratamiento, a través de los informes de los miembros de la familia, proporcionando una mejor calidad de vida a través de una muerte más pacífica sin más sufrimiento.

Palabras clave: Extubación Traqueal; Cuidados Paliativos al Final de la Vida/métodos; Inutilidad Médica; Enfermo Terminal.

${ }^{1}$ Faculdade de Medicina do ABC. Santo André (SP), Brasil. Orcid iD: https://orcid.org/0000-0003-2801-0131

2Universidade Federal de São Paulo (Unifesp). Santo André (SP), Brasil. Orcid iD: https://orcid.org/0000-0002-1873-8148

${ }^{3}$ Faculdade de Medicina do ABC. Santo André (SP), Brasil. Orcid iD: https://orcid.org/0000-0002-7031-3235

Endereço para correspondência: Bianca Kemmilly Rodrigues Paiva. Avenida Príncipe de Gales, 821 - Príncipe de Gales. Santo André (SP), Brasil. CEP 09060-650.

E-mail: biiakemmilly@outlook.com 


\section{INTRODUÇÃO}

O cuidado paliativo tem como objetivo minimizar o sofrimento físico, psicológico, social e espiritual tanto do paciente que enfrenta uma doença que ameaça a vida quanto de seus familiares, por meio de uma abordagem terapêutica multiprofissional ${ }^{1}$.

Para os pacientes que possuem necessidade de tratamento paliativo, este deve ser iniciado o mais precocemente possível, de forma simultânea com o tratamento curativo para melhor alcance dos objetivos, como, por exemplo, proporcionar conforto, controle dos sintomas, qualidade e prolongamento da vida ${ }^{2}$.

As doenças crônicas não transmissíveis (DCNT) são a principal causa de morte no mundo, sendo grande parte com idade abaixo de 70 anos. Estas são consideradas um problema global de saúde, visto a ligação com fatores evitáveis, como sedentarismo e uso de tabaco. Como consequência, projetos nacionais com apoio internacional são estudados e reconhecidos pelos Governos para redução de mortes prematuras ${ }^{3}$.

Segundo as transiçóes demográficas e epidemiológicas globais, o câncer terá um impacto cada vez maior com o passar das décadas ${ }^{4}$. As estimativas do Instituto Nacional de Câncer José Alencar Gomes da Silva (INCA) mostram que, a cada ano do triênio de 2020-2022, ocorrerão 625 mil casos novos de câncer no Brasil. Exceto para o câncer de pele não melanoma (cerca de 177 mil casos novos), os cânceres de mama e próstata (66 mil cada um) e o de cólon e reto (41 mil) serão os mais frequentes na população 5 .

É ciente que, ao conscientizar que as pessoas são seres finitos, o processo de cuidados paliativos aos pacientes terminais sofre alteração. A terminalidade de vida é definida quando se esgotam as possibilidades de resgate das condiçóes de saúde e a possibilidade de morte próxima parece inevitável e previsível. A assistência ao paciente e à família deve contemplar todas as etapas da doença terminal com o objetivo de proporcionar alívio ao sofrimento e evitar medidas fúteis diante da irreversibilidade da doença ${ }^{6}$.

Uma das formas de amenizar o sofrimento dos pacientes terminais é a extubação paliativa, aplicada naqueles em que a morte já é prevista. Esse procedimento evita prolongar a morte e deve ser acordado juntamente com os familiares, de forma que o processo do luto se inicie precocemente ${ }^{7,8}$.

Visto o aumento das DCNT, os cuidados paliativos são de extrema importância para proporcionar uma melhor qualidade de vida para os pacientes e seus familiares, entretanto, a extubação paliativa ainda é pouco difundida. O objetivo deste estudo é realizar uma revisão integrativa e analisar/apresentar o impacto da extubação paliativa em pacientes terminais.

\section{MÉTODO}

A pesquisa foi realizada por meio de busca nas bases de dados: PEDro, PubMed e LILACS, utilizando os descritores: airway extubation, palliative care e hospice care. Como estratégia de busca, foi utilizado o operador booleano AND entre os descritores supracitados.

Os artigos foram analisados pelo título e pelo resumo para obtenção de estudos pertinentes e relevantes para o trabalho, portanto, como critérios de inclusão para a revisão, foram selecionados apenas os artigos que tinham pertinência sobre o tema e analisavam o impacto ou citavam o desfecho da extubação paliativa.

A busca dos artigos foi realizada em junho de 2019. Não foi determinada uma data de corte em razão da baixa quantidade de estudos disponíveis sobre o tema. Os artigos encontrados eram de 2001 a 2019.

\section{RESULTADOS}

$\mathrm{Na}$ base de dados PEDro, buscando os descritores airway extubation e palliative care, e, em seguida, airway extubation e hospice care, não foi encontrado nenhum estudo.

Na base de dados PubMed, selecionando os descritores airway extubation e palliative care, foram encontrados 39 estudos, sendo apenas 13 pertinentes para inclusão na atual pesquisa. Ainda na base de dados PubMed, foi realizada uma busca utilizando os descritores airway extubation e hospice care, na qual foram encontrados quatro estudos, sendo três já inclusos na busca primária, resultando apenas em um novo estudo para contribuição desta pesquisa.

$\mathrm{Na}$ base de dados LILACS, utilizando-se os descritores airway extubation e palliative care, foi encontrado um estudo; já em uma busca secundária com os descritores airway extubation e hospice care, nenhum estudo foi localizado.

Portanto, a atual revisão foi composta de 15 estudos apresentados a seguir (Quadro 1) de acordo com seus respectivos dados.

Os resultados da busca são explicados no fluxograma (Figura 1).

\section{DISCUSSÃO}

A morte passou de um processo agudo para crônico, gerando conflitos sobre a qualidade de vida dos pacientes com uma sobrevida prolongada. Desta forma, com o avanço dos estudos, o cuidado paliativo foi incrementado no tratamento das DCNT, a fim de proporcionar alívio do sofrimento, sendo ele físico ou mental, tanto do doente como de seus familiares. 
Quadro 1. Apresentação dos estudos utilizados

\begin{tabular}{|c|c|c|c|c|c|}
\hline Tí́tulo & Autor/Ano & $\begin{array}{l}\text { Tipo de } \\
\text { estudo }\end{array}$ & Objetivo do estudo & Conclusão do autor & $\begin{array}{c}\text { Impacto da extubação } \\
\text { paliativa }\end{array}$ \\
\hline $\begin{array}{l}\text { Pediatric Critical Care } \\
\text { Transport as a Conduit } \\
\text { to Terminal Extubation } \\
\text { at home: a Case Series }\end{array}$ & $\begin{array}{c}\text { Noje, } \\
\text { Bernier, } \\
\text { Costabile et al. }{ }^{9}, \\
2017\end{array}$ & Estudo de caso & $\begin{array}{l}\text { Apresentar a rotina de três } \\
\text { transportes paliativos para } \\
\text { extubação terminal, em uma } \\
\text { unidade de terapia intensiva } \\
\text { (UTI) pediátrica }\end{array}$ & $\begin{array}{l}\text { A ida para casa, para } \\
\text { realização da extubação } \\
\text { paliativa, é uma alternativa } \\
\text { viável, graças à melhora no } \\
\text { transporte, forma de manejo } \\
\text { e análises de custos }\end{array}$ & $\begin{array}{l}\text { É viável a realização da } \\
\text { extubaçãa paliativa em casa, } \\
\text { mesmo que ocorra uma } \\
\text { apreensão no transporte, } \\
\text { cumprindo assim o desejo dos } \\
\text { familiares de que o fim da vida } \\
\text { seja próximo a eles }\end{array}$ \\
\hline $\begin{array}{l}\text { How Long Does (s)He } \\
\text { Have? Retrospective } \\
\text { Analysis of Outcomes } \\
\text { After Palliative } \\
\text { Extubation in Elderly, } \\
\text { Chronically Critically III } \\
\text { Patients }\end{array}$ & $\begin{array}{l}\text { Pan, Platis, } \\
\text { Maw et al. }{ }^{10} \\
2016\end{array}$ & $\begin{array}{c}\text { Estudo } \\
\text { retrospectivo }\end{array}$ & $\begin{array}{l}\text { Analisar o tempo de vida } \\
\text { pós-extubação paliativa em } \\
\text { pacientes crônicos idosos }\end{array}$ & $\begin{array}{l}\text { Grande parte dos pacientes } \\
\text { que participaram do estudo } \\
\text { foi a óbito após a extubação } \\
\text { paliativa no próprio hospital, } \\
\text { sendo que a grande maioria } \\
\text { sobreviveu sem ventilador, } \\
\text { em média, } 24 \text { horas }\end{array}$ & $\begin{array}{l}\text { Por mais que grande parte dos } \\
\text { pacientes tenha ido a óbito no } \\
\text { próprio hospital, percebe-se } \\
\text { uma considerável chance de o } \\
\text { paciente ir para a casa e vir a } \\
\text { óbito após um tempo }\end{array}$ \\
\hline $\begin{array}{l}\text { Respiratory Therapists' } \\
\text { Experiences and } \\
\text { Attitudes Regarding } \\
\text { Terminal Extubations } \\
\text { and End-of-life Care }\end{array}$ & $\begin{array}{c}\text { Grandhige, } \\
\text { Timmer, } \\
\text { 0'Neill et al." } \\
2016\end{array}$ & Estudo de caso & $\begin{array}{l}\text { Analisar a experiência dos } \\
\text { terapeutas respiratórios, } \\
\text { em dois centros médicos } \\
\text { acadêmicos, com pacientes que } \\
\text { realizariam extubação paliativa }\end{array}$ & $\begin{array}{l}\text { Raramente, os } \\
\text { fisioterapeutas respiratórios } \\
\text { estão envolvidos nas } \\
\text { reuniões sobre os pacientes } \\
\text { terminais. Há necessidade } \\
\text { de um treinamento e melhor } \\
\text { relação entre equipe- } \\
\text {-paciente }\end{array}$ & $\begin{array}{l}\text { Os sintomas pós-extubação } \\
\text { paliativa poderiam ser mais bem } \\
\text { controlados, se os fisioterapeutas } \\
\text { respiratórios tivessem auxílio } \\
\text { dos demais profissionais, como } \\
\text { médicos e enfermeiras }\end{array}$ \\
\hline $\begin{array}{l}\text { Compassionate } \\
\text { Extubation for a } \\
\text { Peaceful Death in the } \\
\text { Setting of a Community } \\
\text { Hospital: a Case-Series } \\
\text { Study }\end{array}$ & Kok $^{12}, 2015$ & Estudo de caso & $\begin{array}{l}\text { Apresentar a experiência em } \\
\text { um hospital universitário sobre } \\
\text { o tempo de vida dos pacientes } \\
\text { pós-extubação, uso de sedação } \\
\text { paliativa, local para realizar } \\
\text { a extubação, quem realiza } \\
\text { e quanto tempo leva para } \\
\text { realizar todo o processo, desde } \\
\text { a decisão até o momento exato } \\
\text { da extubação }\end{array}$ & $\begin{array}{l}0 \text { tempo de morte varia } \\
\text { de acordo com a doença } \\
\text { existente, sendo em média } \\
0,3 \text { hora em pacientes } \\
\text { com parada cardíaca fora } \\
\text { do hospital e } 97 \text { horas } \\
\text { em pacientes em estágios } \\
\text { avançados de câncer }\end{array}$ & $\begin{array}{l}\text { A qualidade da morte é de } \\
\text { extrema importância, pois } \\
\text { discute-se que nem todos os } \\
\text { pacientes que realizarão a } \\
\text { extubação paliativa necessitam } \\
\text { efetivamente de sedação. Há } \\
\text { necessidade apenas para aqueles } \\
\text { que já fazem uso de morfina } \\
\text { para controle da dor }\end{array}$ \\
\hline $\begin{array}{l}\text { Your Own Sweet Time: } \\
\text { Discontinuing Ventilator } \\
\text { Support at Home }\end{array}$ & $Y_{\text {eow }}{ }^{13}, 2015$ & Relato de caso & $\begin{array}{l}\text { Relatar o retorno de um } \\
\text { paciente terminal para casa, } \\
\text { por desejo de seus familiares, } \\
\text { a fim de realizar a extubação } \\
\text { paliativa domiciliar }\end{array}$ & $\begin{array}{l}\text { Percebeu-se que a família } \\
\text { optou pelo alívio do } \\
\text { sofrimento de seu filho, no } \\
\text { lugar que ele sempre sentiu } \\
\text { feliz. E que, no início, os } \\
\text { familiares se mostram mais } \\
\text { desconfortáveis com o ato de } \\
\text { se despedir, mas depois se } \\
\text { torna um momento de paz } \\
\text { para todos }\end{array}$ & $\begin{array}{l}\text { A família demonstrou todo o } \\
\text { amor ao paciente, retornando } \\
\text { para o lugar onde ele sempre foi } \\
\text { feliz e ao lado de todos os seus } \\
\text { familiares que se despediram } \\
\text { pela última vez }\end{array}$ \\
\hline $\begin{array}{l}\text { Retirada de asistencia } \\
\text { respiratoria en } \\
\text { domicilio: toma de } \\
\text { decisiones en cuidados } \\
\text { paliativos pediátricos }\end{array}$ & $\begin{array}{l}\text { García-Salido, } \\
\text { Monleón- } \\
\text { Luque, } \\
\text { Barceló-Escario } \\
\text { et al. }^{14}, 2014\end{array}$ & Relato de caso & $\begin{array}{l}\text { Relatar o retorno de um } \\
\text { paciente terminal para a } \\
\text { realização da extubação } \\
\text { paliativa, a fim de aumentar } \\
\text { o vínculo familiar na fase final } \\
\text { da vida }\end{array}$ & $\begin{array}{l}\text { Percebe-se que o melhor } \\
\text { ambiente para o processo de } \\
\text { extubaçãa e a comunicação } \\
\text { da equipe com os familiares } \\
\text { é no ambiente domiciliar, de } \\
\text { fato que este se apresenta } \\
\text { menos barulhento, frio e } \\
\text { angustiante do que uma UTI }\end{array}$ & $\begin{array}{l}\text { É de extrema importância a } \\
\text { relação direta da equipe de } \\
\text { cuidados paliativos e a família } \\
\text { para melhor conforto tanto } \\
\text { do paciente quanto de seus } \\
\text { familiares no momento da } \\
\text { extubação }\end{array}$ \\
\hline $\begin{array}{l}\text { Transport Home and } \\
\text { Terminal Extubation } \\
\text { by Emergency Medical } \\
\text { Services: an Example of } \\
\text { Innovation in End-of-life } \\
\text { Care }\end{array}$ & $\begin{array}{l}\text { Clemency, } \\
\text { Grimm, } \\
\text { Lauer et al. }^{15} \text {, } \\
2019\end{array}$ & Relato de caso & $\begin{array}{l}\text { Descrever uma extubação } \\
\text { terminal realizada por um } \\
\text { paramédico sob a supervisão } \\
\text { direta de um médico dos } \\
\text { serviços médicos de emergência } \\
\text { na casa do paciente }\end{array}$ & $\begin{array}{l}\text { Há a possibilidade de } \\
\text { realização da extubação } \\
\text { paliativa em âmbito } \\
\text { domiciliar, basta que esta } \\
\text { seja uma escolha da família } \\
\text { e do paciente em questão }\end{array}$ & $\begin{array}{l}\text { Para alcançar verdadeiramente } \\
\text { o atendimento centrado no } \\
\text { paciente no final da vida, é } \\
\text { necessária a escolha de uma } \\
\text { morte fora do hospital }\end{array}$ \\
\hline
\end{tabular}


Quadro 1. continuação

\begin{tabular}{|c|c|c|c|c|c|}
\hline Tí́tulo & Autor/Ano & $\begin{array}{l}\text { Tipo de } \\
\text { estudo }\end{array}$ & Objetivo do estudo & Conclusão do autor & $\begin{array}{c}\text { Impacto da extubação } \\
\text { paliativa }\end{array}$ \\
\hline $\begin{array}{l}\text { Ventilator Withdrawal: } \\
\text { Procedures and } \\
\text { Outcomes. Report of a } \\
\text { Collaboration Between } \\
\text { a Critical Care Division } \\
\text { and a Palliative Care } \\
\text { Service }\end{array}$ & $\begin{array}{l}\text { O'Mahony, } \\
\text { McHugh, } \\
\text { Zallman et al. }{ }^{16}, \\
2003\end{array}$ & Estudo de caso & $\begin{array}{l}\text { Descrever o procedimento de } \\
\text { extubação paliativa e analisar } \\
\text { as respostas apresentadas pelos } \\
\text { pacientes }\end{array}$ & $\begin{array}{l}\text { Conclui-se que as famílias } \\
\text { geralmente sabem pouco } \\
\text { sobre a importância dos } \\
\text { cuidados paliativos e, desta } \\
\text { forma, o processo acaba se } \\
\text { tornando mais demorado }\end{array}$ & $\begin{array}{l}\text { Não é de acordo apresentar } \\
\text { um prognóstico fixo para a } \\
\text { família dos pacientes, pois, } \\
\text { como apresentado no artigo, } \\
\text { houve uma porcentagem que } \\
\text { teve continuidade nos cuidados } \\
\text { paliativos pós-extubação }\end{array}$ \\
\hline $\begin{array}{c}\text { Withdrawal of } \\
\text { Ventilatory support } \\
\text { outside the intensive } \\
\text { care unit: guidance for } \\
\text { practice }\end{array}$ & $\begin{array}{l}\text { Laddie, } \\
\text { Craig, } \\
\text { Brierley et al. }{ }^{17}, \\
2014\end{array}$ & $\begin{array}{c}\text { Estudo } \\
\text { transversal }\end{array}$ & $\begin{array}{l}\text { Revisar o estudo de um } \\
\text { pediatra em cuidados paliativos } \\
\text { que realizou a retirada do } \\
\text { suporte ventilatório fora da } \\
\text { UTI, a fim de melhorar as } \\
\text { orientações para tal prática }\end{array}$ & $\begin{array}{l}\text { Conclui-se que a retirada do } \\
\text { suporte ventilatório fora da } \\
\text { UTI é desafiador e necessita } \\
\text { de diversos recursos. E } \\
\text { como a realização de novos } \\
\text { estudos auxilia na decisãa } \\
\text { dos familiares com relação } \\
\text { ao local preferido para a } \\
\text { extubação ser realizada }\end{array}$ & $\begin{array}{l}\text { Todos os pacientes que foram } \\
\text { submetidos à extubação paliativa } \\
\text { estavam com seus familiares } \\
\text { presentes e tiveram variações } \\
\text { com relação ao tempo de morte. } \\
\text { São cruciais para a eficácia do } \\
\text { procedimento a comunicação } \\
\text { e o planejamento da equipe } \\
\text { juntamente com os familiares }\end{array}$ \\
\hline $\begin{array}{l}\text { Pediatric Extubation: } \\
\text { "Pulling the Tube" }\end{array}$ & $\begin{array}{l}\text { Sine, Sumner, } \\
\text { Gracy et al. }{ }^{18} \text {, } \\
2001\end{array}$ & Relato de caso & $\begin{array}{l}\text { Relatar momentos antes } \\
\text { da extubação paliativa e a } \\
\text { importância de uma boa } \\
\text { abordagem da equipe com os } \\
\text { familiares }\end{array}$ & $\begin{array}{l}\text { Cada local precisa } \\
\text { desenvolver uma forma de } \\
\text { atender às necessidades } \\
\text { de cada criança, família e } \\
\text { equipe. Cada indivíduo, } \\
\text { independentemente da } \\
\text { idade ou doença, tem direito } \\
\text { a uma morte digna }\end{array}$ & $\begin{array}{l}\text { A família impõe um papel } \\
\text { extremamente importante na } \\
\text { decisão, pois devem acreditar } \\
\text { que estão prevenindo um } \\
\text { sofrimento ao seu filho }\end{array}$ \\
\hline $\begin{array}{l}\text { Interdisciplinary } \\
\text { Pediatric Palliative Care } \\
\text { Team Involvement } \\
\text { in Compassionate } \\
\text { Extubation at Home: } \\
\text { From Shared Decision- } \\
\text { Making to Bereavement }\end{array}$ & $\begin{array}{l}\text { Postier, Catrine, } \\
\text { Remke }^{19}, 2018\end{array}$ & $\begin{array}{c}\text { Relato de série } \\
\text { de caso }\end{array}$ & $\begin{array}{l}\text { Demonstrar a importância da } \\
\text { equipe de cuidados paliativos } \\
\text { pediátricos em dois casos }\end{array}$ & $\begin{array}{l}\text { Conclui-se que é de extrema } \\
\text { importância a presença de } \\
\text { uma equipe interdisciplinar } \\
\text { que constitui a equipe de } \\
\text { cuidados paliativos }\end{array}$ & $\begin{array}{l}\text { Demonstrou a dificuldade no } \\
\text { transporte para uma área rural } \\
\text { de difícil acesso, prejudicando } \\
\text { também à chegada dos médicos } \\
\text { pós-extubação, uma vez que o } \\
\text { paciente sobreviveu } 2 \text { dias, o que } \\
\text { não era esperado pela equipe }\end{array}$ \\
\hline $\begin{array}{l}\text { Factors associated with } \\
\text { palliative withdrawal of } \\
\text { mechanical ventilation } \\
\text { and time to death after } \\
\text { withdrawal }\end{array}$ & $\begin{array}{l}\text { Huynh, Walling, } \\
\text { Le et al. }{ }^{7}, 2013\end{array}$ & $\begin{array}{l}\text { Estudo } \\
\text { retrospectivo }\end{array}$ & $\begin{array}{l}\text { Identificar fatores associados à } \\
\text { extubação paliativa e o tempo } \\
\text { até a morte pós-extubação }\end{array}$ & $\begin{array}{l}\text { Conclui-se que pacientes } \\
\text { mais dependentes de terapia } \\
\text { de suporte (com Fi02 } \\
\text { maiores que } 70 \% \text { e o uso } \\
\text { de vasopressores) levam um } \\
\text { tempo menor até o óbito }\end{array}$ & $\begin{array}{l}\text { Os pacientes presentes nesse } \\
\text { estudo sobreviveram no máximo } \\
165 \text { dias pós-extubação. Porém, } \\
\text { a maioria sobreviveu } 10 \text { horas } \\
\text { após o procedimento }\end{array}$ \\
\hline $\begin{array}{l}\text { Survey of Respiratory } \\
\text { Therapists' Attitudes } \\
\text { and Concerns Regarding } \\
\text { Terminal Extubation }\end{array}$ & $\begin{array}{l}\text { Willms e } \\
\text { Brewer }^{20} \\
2005\end{array}$ & $\begin{array}{c}\text { Estudo de } \\
\text { monitoramento }\end{array}$ & $\begin{array}{l}\text { Identificar a extensão } \\
\text { do envolvimento dos } \\
\text { fisioterapeutas respiratórios no } \\
\text { processo de extubação paliativa }\end{array}$ & $\begin{array}{l}\text { A maioria dos entrevistados } \\
\text { participou efetivamente } \\
\text { da extubação paliativa e } \\
\text { acredita que a presença } \\
\text { do médico é de extrema } \\
\text { importância, gerando assim } \\
\text { uma padronização no } \\
\text { procedimento }\end{array}$ & $\begin{array}{l}\text { Os fisioterapeutas respiratórios } \\
\text { entrevistados no presente estudo } \\
\text { acreditam que o papel deles } \\
\text { na extubação paliativa é o de } \\
\text { garantir a qualidade de vida, } \\
\text { seguido por gerar alívio do } \\
\text { sofrimento }\end{array}$ \\
\hline $\begin{array}{c}\text { Home Extubation in a } \\
\text { Neonate }\end{array}$ & $\begin{array}{l}\text { Loganathan, } \\
\text { Simpson, } \\
\text { Boutcher et } \\
\text { a. }{ }^{21}, 2018\end{array}$ & Relato de caso & $\begin{array}{l}\text { Relatar um caso em que foi } \\
\text { realizada a extubação em } \\
\text { ambiente domiciliar e explicar } \\
\text { os processos envolvidos }\end{array}$ & $\begin{array}{l}\text { Conclui-se que os médicos } \\
\text { concordam que a ida para } \\
\text { casa é o melhor caminho, } \\
\text { porém acreditam que } \\
\text { necessitem de mais recursos } \\
\text { para melhor efetividade. } \\
\text { Também se percebe que o } \\
\text { processo de luto é maior nos } \\
\text { mais daqueles que foram a } \\
\text { óbito em um leito de UTI do } \\
\text { que aqueles que morreram } \\
\text { em casa }\end{array}$ & $\begin{array}{l}\text { A extubação foi realizada no colo } \\
\text { da mãe, e o bebê veio a óbito } \\
\text { horas depois do procedimento. } \\
\text { Após } 6 \text { semanas, a equipe se } \\
\text { encontrou com a família, a qual } \\
\text { demonstrou imensa gratidão } \\
\text { pela oportunidade de passar um } \\
\text { tempo com seu filho em casa } \\
\text { antes do óbito }\end{array}$ \\
\hline
\end{tabular}


Quadro 1. continuação

\begin{tabular}{|c|c|c|c|c|c|}
\hline Título & Autor/Ano & $\begin{array}{l}\text { Tipo de } \\
\text { estudo }\end{array}$ & Objetivo do estudo & Conclusão do autor & $\begin{array}{c}\text { Impacto da exłubação } \\
\text { paliativa }\end{array}$ \\
\hline $\begin{array}{l}\text { Palliative Extubation: } \\
\text { Case Analysis in an } \\
\text { Intensive Care Unit }\end{array}$ & $\begin{array}{c}\text { Rebelatto e } \\
\text { Moritz }^{22}, 2017\end{array}$ & $\begin{array}{l}\text { Estudo de } \\
\text { coorte }\end{array}$ & $\begin{array}{c}\text { Avaliar o perfil } \\
\text { clinicodemográfico de pacientes } \\
\text { extubados paliativamente } \\
\text { na UTI de um hospital } \\
\text { universitário }\end{array}$ & $\begin{array}{l}\text { Conclui-se que a maior parte } \\
\text { dos pacientes que foram } \\
\text { submetidos à extubação } \\
\text { paliativa eram idosos, } \\
\text { acometidos por doenças } \\
\text { neurológicas e tiveram um } \\
\text { intervalo entre o momento } \\
\text { da extubação e o óbito de } \\
\text { em média } 2,5 \text { dias }\end{array}$ & $\begin{array}{l}\text { Todos os pacientes no presente } \\
\text { estudo estavam na UTI sob efeito } \\
\text { de analgésicos no momento da } \\
\text { extubação. Foi constatado que os } \\
\text { pacientes sobrevivem de minutos } \\
\text { a dias, porém mais da metade } \\
\text { vieram a óbito após } 24 \text { horas do } \\
\text { procedimento. Acredita-se que } \\
\text { o tempo de vida pós-extubação } \\
\text { está relacionado à gravidade } \\
\text { da doença e não à retirada do } \\
\text { ventilador }\end{array}$ \\
\hline
\end{tabular}

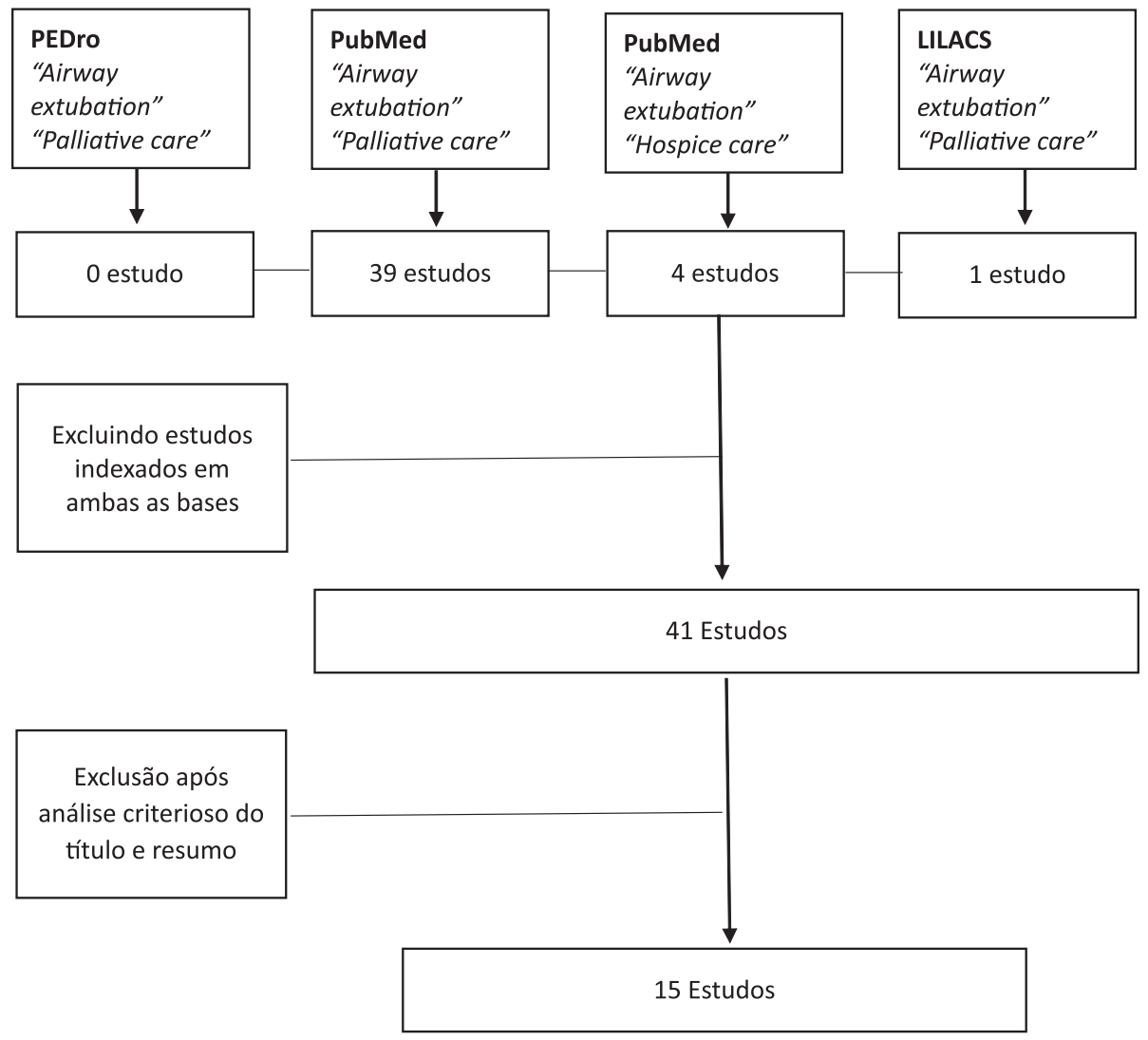

Figura 1. Fluxograma dos estudos identificados

Uma das formas de proporcionar o alívio é a retirada de medidas invasivas, como a ventilação mecânica. Nos pacientes cuja morte já é esperada, opta-se pela extubação paliativa, a qual foi analisada no presente estudo quanto ao local de realização, ao tempo de vida pós-procedimento, ao uso de sedação, entre outros.

Geralmente, as famílias pouco sabem sobre a importância dos cuidados paliativos, tornando o processo mais demorado ${ }^{16}$. Como a família impóe um papel de extrema importância na decisão, uma boa equipe e um local que atenda às necessidades de todos são cruciais para uma morte tranquila para o paciente, amenizando o sofrimento de todos ${ }^{18}$.

Segundo estudo realizado na unidade de terapia intensiva (UTI) de um Hospital Universitário, grande parte dos pacientes, que foram submetidos à extubação paliativa, é de idosos, acometidos por doenças neurológicas, tendo como tempo de vida pós-procedimento variável de minutos a dias, porém sua maioria foi a óbito após 24 horas. Rebelatto e Moritz ${ }^{22}$ acreditam que o tempo de vida 
pós-extubação está relacionado à gravidade da doença, e não à retirada do suporte ventilatório.

Já subsequente a este estudo, em outra pesquisa, observou-se que grande parte dos pacientes analisados foi a óbito no local onde foi realizada a extubação paliativa, no próprio hospital. Sendo que a maioria dos pacientes também sobreviveram em média 24 horas após o procedimento, fato condizente com o estudo anteriormente analisado ${ }^{10}$.

Pacientes que são dependentes de terapia de suporte, como ventilaçóes com $\mathrm{FiO} 2$ maiores que $70 \%$ e uso de vasopressores, levam um tempo menor até o óbito após a extubação. Neste estudo, os pacientes sobreviveram no máximo 165 dias, porém sua maioria não ultrapassou as 10 horas de vida após o procedimento ${ }^{7}$. Já para $\mathrm{Kok}^{12}$, o tempo de morte é variável de acordo com a doença existente, sendo que, no estudo realizado, variou de 0,3 hora (levando o paciente a uma parada cardíaca) até 97 horas (em pacientes em estágios avançados de câncer). Também foi discutido que a qualidade da morte é de extrema importância, então, houve sedação no momento da extubação apenas naqueles pacientes que já faziam uso de algum medicamento para dor, como, por exemplo, a morfina.

Para a melhor qualidade da morte, Truog et al. ${ }^{23}$ descreveram que todos os pacientes devem receber sedaçáo antecipatória antes do procedimento, a fim de evitar sofrimento e dor a esses pacientes, visto que não há a necessidade de aguardar um sinal de desconforto, para a administração de sedativos, pois, desta forma, poderia causar mais sofrimento para o paciente e seus familiares.

Além do uso de sedação ou não, é discutível a retirada do tubo orotraqueal desses pacientes. Matzo e Orwig ${ }^{24}$ analisaram que essa retirada não possui um protocolo, pois é variável de acordo com cada situaçáo, valores, crenças e conforto do paciente. Alguns pacientes podem desenvolver edema de glote, hipersecreção ou obstrução das vias aéreas, fatores que podem levar a um desconforto respiratório após a extubação. Já fatores como estética facial para uma última despedida de seus familiares levam à retirada do tubo, proporcionando um último momento com melhores lembranças visuais do paciente. Então, pela presença de poucos estudos, deve-se analisar o melhor benefício para o paciente, para que este não apresente nenhum sofrimento.

Para Grandhige et al. ${ }^{11}$, os fisioterapeutas respiratórios raramente são envolvidos nas reunióes de equipe e dos familiares, então, acredita-se que, por tal motivo, esse profissional está pouco inserido nesse processo. E muitos dos sintomas encontrados no ato da extubaçáo, como desconforto respiratório, poderiam ser evitados.

Em contrapartida, Willms e Brewer ${ }^{20}$ mostraram que a maioria dos fisioterapeutas respiratórios participou do procedimento e acham de extrema importância, tornando um atendimento mais padronizado. Os profissionais acreditam que a sua presença melhora a qualidade de vida do paciente, gerando alívio do sofrimento.

De forma geral, o retorno de um paciente terminal para casa é bem relatado nos estudos, por ser menos barulhento, frio e angustiante do que uma $\mathrm{UTI}^{14}$. Mas, apesar de estudos relatarem o domicílio como melhor local, um estudo transversal mostra que esse procedimento fora da UTI é extremamente desafiador, necessitando de diversos recursos e novos estudos para melhorar a prática ${ }^{17}$.

Além disso, um estudo mais recente de Clemency et al. ${ }^{15}$ comprovou a possibilidade de realização da extubação paliativa em casa, desde que seja uma opçáo dos familiares e do próprio paciente, se possível. A ida para casa só se tornou uma alternativa viável após a melhoria do transporte, a forma de manejo desse paciente e análise de custos (UTI e domicílio), mesmo que haja apreensão dos profissionais e dos familiares no transporte até a residência do paciente?

Concomitantemente aos demais estudos que analisaram o benefício dá ida para casa para o paciente e os familiares, Loganathan et al. ${ }^{21}$ também relataram ser a melhor escolha, mas acreditam que necessite de mais recursos para melhor efetividade do procedimento. Esse estudo também relata que o processo de luto dos familiares dentro de uma UTI se torna maior quando comparados com aqueles familiares que acompanharam a morte do paciente em seus domicílios. Mesmo com todos os benefícios de uma extubação paliativa domiciliar, como também relatado no estudo de Yeow ${ }^{13}$, que demonstrou um momento de paz para todos os familiares, pois o paciente foi levado para o local onde ele foi feliz.

Em um relato de caso de Postier et al. ${ }^{19}$, houve dificuldade no transporte para o domicílio de um paciente que residia na área rural e, mesmo sem possuir prognóstico de vida após a extubaçáo, sobreviveu dois dias, sendo o local de difícil acesso para a equipe de saúde.

\section{CONCLUSÃO}

Tendo em vista o aumento dos pacientes com DCNT, é evidente que os cuidados paliativos são de extrema importância para eles, a fim de proporcionar melhora da qualidade de vida tanto do doente como de seus familiares.

Diante dessa análise, foi observado, de acordo com relatos dos familiares, que a extubação paliativa é para todos a melhor opçáa, pois torna o fim da vida mais sutil, diminuindo o sofrimento de uma forma geral.

Diante da contextualização apresentada e tendo em vista a importância da extubação paliativa, sugere-se a realização de novas pesquisas que avaliem os efeitos e os 
possíveis protocolos desse processo. Desse modo, esta pesquisa contribuirá para a construção de protocolos sobre o tema e para a efetividade da indicação e da aplicação dessas terapêuticas no manejo dos cuidados paliativos.

\section{CONTRIBUIÇÕES}

Beatriz Louro contribuiu substancialmente na elaboração, concepção e/ou planejamento do estudo; na obtenção, análise e interpretação dos dados; assim como na revisão crítica. Bianca Kemmilly Rodrigues Paiva contribuiu na revisão crítica. Amanda Estevão contribuiu substancialmente na elaboração, concepção e/ou planejamento do estudo; na obtenção, análise e interpretação dos dados; assim como na redação. Todas as autoras aprovaram a versão final a ser publicada.

\section{FONTES DE FINANCIAMENTO}

Não há.

\section{DECLARAÇÃO DE CONFLITO DE INTERESSES}

Nada a declarar.

\section{REFERÊNCIAS}

1. Oliveira RA, coordenador. Cuidado Paliativo [Internet]. São Paulo: CREMESP; 2008. Capítulo 1, Maciel, MGS. Definiçôes e princípios [acesso 2020 abr 10]; p. 15-32. Disponível em: https://edisciplinas.usp.br/pluginfile. php/446028/mod_resource/content/1/Cuidados_ Paliativos_CREMESP.pdf

2. World Health Organization. National cancer control programmes: policies and managerial guidelines [Internet]. 2nd ed. Geneva: WHO; 2002 [cited |2020 Apr 10]. Available from: https://apps.who.int/ iris/bitstream/handle/10665/42494/9241545577. pdf?sequence $=1$ \&isAllowed $=y$

3. World Health Organization [Internet]. Geneva: WHO; c2020. Global Action plan for the prevention and control of NCDs 2013-2020; [2013] [cited 2019 May 15]. Available from: https://www.who.int/nmh/events/ ncd_action_plan/en/

4. Ferlay J, Colombet M, Soerjomataram I, et al. Estimating the global cancer incidence and mortality in 2018: GLOBOCAN sources and methods. Int J Cancer. 2019;144(8):1941-53. doi: https://doi.org/10.1002/ ijc.31937

5. Instituto Nacional de Câncer José Alencar Gomes da Silva. Estimativa 2020: incidência de câncer no Brasil [Internet]. Rio de Janeiro: INCA; 2019 [acesso 2020 out 27. Disponível em: https://www.inca.gov.br/publicacoes/ livros/estimativa-2020-incidencia-de-cancer-no-brasil
6. Marengo MO, Flávio DA, Silva RHA. Terminalidade de vida: bioética e humanização. Med (Ribeirão Preto). 2009; 42(3):350-7. doi: https://doi.org/10.11606/ issn.2176-7262.v42i3p350-357

7. Huynh TN, Walling AM, Le TX, et al. Factors associated with palliative withdrawal of mechanical ventilation and time to death after withdrawal. J Palliat Med. 2013;16(11):1368-74. doi: https://doi.org/10.1089/ jpm.2013.0142

8. Lage JSS, Pincelli ASM, Furlan JAS, et al. Extubação paliativa em unidade de emergência: relato de caso. Rev Bioét. 2019;27(2):313-7. doi: http://doi. org/10.1590/1983-80422019272315.

9. Noje C, Bernier ML, Costabile PM, et al. Pediatric critical care transport as a conduit to terminal extubation at home: a case series. Pediatr Crit Care Med. 2017;18(1):e4-ee8. doi: http://doi.org/10.1097/PCC.0000000000000997

10. Pan CX, Platis D, Maw MM, et al. How long does (s)he have? retrospective analysis of outcomes after palliative extubation in elderly, chronically critically III patients. Crit Care Med. 2016;44(6):1138-44. doi: http://doi. org/10.1097/CCM.0000000000001642

11. Grandhige AP, Timmer M, O’Neill MJ, et al. Respiratory therapists' experiences and attitudes regarding terminal extubations and end-of-life care. Respir Care. 2016;61(7):891-6. doi: http://doi.org/10.4187/ respcare. 04168

12. Kok VC. Compassionate extubation for a peaceful death in the setting of a community hospital: a case-series study. Clin Interv Aging. 2015;10:679-85. doi: http:// doi.org/10.2147/CIA.S82760

13. Yeow ME. Your own sweet time: discontinuing ventilator support at home. J Palliat Med. 2015;18(4):388-9. doi: http://doi.org/10.1089/jpm.2014.0317

14. García-Salido A, Monleón-Luque M, Barceló-Escario $\mathrm{M}$, et al. Retirada de asistencia respiratoria en domicilio: toma de decisiones en cuidados paliativos pediátricos. An Pediatr. 2014;80(3):181-3. doi: http://doi.org/10.1016/j. anpedi.2013.05.007

15. Clemency BM, Grimm KT, Lauer SL, et al. Transport home and terminal extubation by emergency medical services: an example of innovation in end-of-life care. J Pain Symptom Manage. 2019;58(2):355-9. doi: http:// doi.org/10.1016/j.jpainsymman.2019.03.007

16. O’Mahony S, McHugh M, Zallman L, et al. Ventilator withdrawal: procedures and outcomes. Report of a collaboration between a critical care division and a palliative care service. J Pain Symptom Manage. 2003;26(4):954-61. doi: https://doi.org/10.1016/ S0885-3924(03)00333-6

17. Laddie J, Craig F, Brierley J, et al. Withdrawal of ventilatory support outside the intensive care unit: guidance for practice. Arch Dis Child. 2014;99(9):812-6. doi: http://doi.org/10.1136/archdischild-2013-305492 
18. Sine D, Sumner L, Gracy D, et al. Pediatric extubation: "pulling the tube". J Palliat Med. 2001;4(4):519. doi: https://doi.org/10.1089/109662101753381692

19. Postier A, Catrine K, Remke S. Interdisciplinary pediatric palliative care team involvement in compassionate extubation at home: from shared decision-making to bereavement. Children (Bassel). 2018;5(3):37. doi: https://doi.org/10.3390/children5030037

20. Willms D, Brewer JA. Survey of respiratory therapists' attitudes and concerns regarding terminal extubation. Respir Care. 2005 [cited 2019 Oct 11];50(8):1046-9. Available from: http://rc.rcjournal. com/content/50/8/1046/tab-pdf

21. Loganathan P, Simpson J, Boutcher P, et al. Home extubation in a neonate. Pediatrics. 2018;142(1):e20172845. doi: https://doi.org/10.1542/peds.2017-2845

22. Rebelatto G, Moritz RD. Palliative extubation: case analysis in an intensive care unit. Mundo Saúde. 2017;41(3):385-94. doi: https://doi.org/10.15343/01047809.20174103385394

23. Truog RD, Brock DW, White DB. Should patients receive general anesthesia prior to extubation at the end of life? Crit Care Med. 2012;40(2):631-3. doi: https:// doi.org/10.1097/ccm.0b013e3182413b8a

24. Matzo M, Orwig S. 50 shades of gray. J Palliat Med. 2013;16(8):833. doi: https://doi.org/10.1089/ jpm.2013.0185 